\title{
Re-flaring of a Post-Flare Loop System Driven by Flux Rope Emergence and Twisting
}

\author{
X. Cheng ${ }^{1,2}$, M. D. Ding ${ }^{1,2}$, Y. Guo ${ }^{1,2}$, J. Zhang ${ }^{1,3}$, J. Jing ${ }^{4}$, and T. Wiegelmann ${ }^{5}$ \\ 1 Department of Astronomy, Nanjing University, Nanjing 210093, China \\ dmd@nju.edu.cn \\ ${ }^{2}$ Key Laboratory of Modern Astronomy and Astrophysics (Nanjing University), Ministry of \\ Education, Nanjing 210093, China \\ 3 Department of Computational and Data Sciences, George Mason University, 4400 University \\ Drive, MSN 6A2, Fairfax, VA 22030, USA \\ ${ }^{4}$ Space Weather Research Laboratory, New Jersey Institute of Technology, Newark, NJ 07102, \\ $U S A$ \\ ${ }^{5}$ Max-Planck-Institut für Sonnensystemforschung, Max-Planck-Strasse 2, 37191 \\ Katlenburg-Lindau, Germany
}

\begin{abstract}
In this letter, we study in detail the evolution of the post-flare loops on 2005 January 15 that occurred between two consecutive solar eruption events, both of which generated a fast halo CME and a major flare. The post-flare loop system, formed after the first $\mathrm{CME} /$ flare eruption, evolved rapidly, as manifested by the unusual accelerating rise motion of the loops. Through nonlinear force-free field (NLFFF) models, we obtain the magnetic structure over the active region. It clearly shows that the flux rope below the loops also kept rising accompanied with increasing twist and length. Finally, the postflare magnetic configuration evolved to a state that resulted in the second CME/flare eruption. This is an event in which the post-flare loops can re-flare in a short period of $\sim 16 \mathrm{hr}$ following the first $\mathrm{CME} /$ flare eruption. The observed re-flaring at the same location is likely driven by the rapid evolution of the flux rope caused by the magnetic flux emergence and the rotation of the sunspot. This observation provides valuable information on CME/flare models and their prediction.
\end{abstract}

Subject headings: Sun: corona — Sun: coronal mass ejections (CMEs) — Sun: flares — Sun: magnetic topology

\section{Introduction}

The developed systems of loops, named post-flare loops, usually occur in the decay phase of long duration events (e.g., Bruzek 1964; Kahlen 1977; Harra-Murnion et al. 1998). It is generally 
believed that magnetic reconnection plays an important role in the formation and evolution of the post-flare loops. The rising of post-flare loops in the corona, as well as the separation of flare ribbons at the footpoints, can be well explained by the classical reconnection model, i.e., the CSHKP model (Carmichael 1964; Sturrock 1966; Hiravama 1974; Kopp \& Pneuman 1976). In this model, the observed motions are caused by the systematic ascending of the reconnection site in the corona (e.g., Cheng et al. 2010a). The post-flare loops comprise of a system of loops heated and formed consecutively through the ongoing reconnection process. Based on the CSHKP model, the postflare loops rise with a gradually decreasing speed. However, Švestka (1996) found some exceptional cases, in which the post-flare loops rose with a constant speed for a long period of time, and was explained through the combination of two entirely different processes: the initial development of the magnetic reconnection proposed by Kopp \& Pneuman (1976) and the subsequent expanding motion into the corona. It is generally believed that the post-flare loops evolve without further energy input after their formation. Therefore, they usually fade away into the background after a certain time. To our knowledge, there is no report on the re-activation of a post-flare loop system in previous studies.

Coronal mass ejections (CMEs) are another kind of large-scale solar activity that may involve the magnetic field lines that twisted each other, named flux rope, in their core, as revealed by many coronagraph observations (e.g., Pick et al. 2006). The role of flux ropes in the CME eruptions has also been studied extensively through numerical simulation. Amari et al. (2000) simulated the evolution of a flux rope and found that it plays a crucial role in the process of the CME eruption. The flux rope was also used by Fan (2001) and Fan \& Gibson (2003, 2004) to investigate the dynamic evolution of the coronal magnetic field in response to the emergence of twisted magnetic structures. Moreover, Török \& Kliem (2003, 2005) and Kliem \& Török (2006) studied the instability of the flux rope and proposed that the CME eruption can be initiated through the kink and/or torus instability. Cheng et al. (2010b) suggested that the flux rope may provide a magnetic structure favorable for the eruption and regarded the formation period of the flux rope prior to the eruption as the build-up phase of CMEs (see also Su et al. 2009; Aulanier et al. 2010). Schrijver et al. (2008) and Schrijver (2009) showed that the emergence of current-carrying flux ropes can lead to the occurrence of a series of major flares within a few hours in the same active region. Further, Canou et al. (2009) constructed a flux rope structure above magnetic polarity inversion line (PIL) of an emerging sunspot by a nonlinear force-free field (NLFFF) model using the THEMIS vector magnetogram as the input. Recently, Guo et al. (2010) also obtained a flux rope structure through NLFFF modeling and found that the flux rope coincided with the active region filament. However, up to now, there is still no study on the time evolution of extrapolated flux ropes.

In this letter, we report the re-flaring of a post-flare loop system occurred on 2005 January 15, and further study the temporal development of associated flux rope. In section 2, we present the observations and data analysis method. Our results are shown in section 3 followed by summary and discussions in section 4 . 

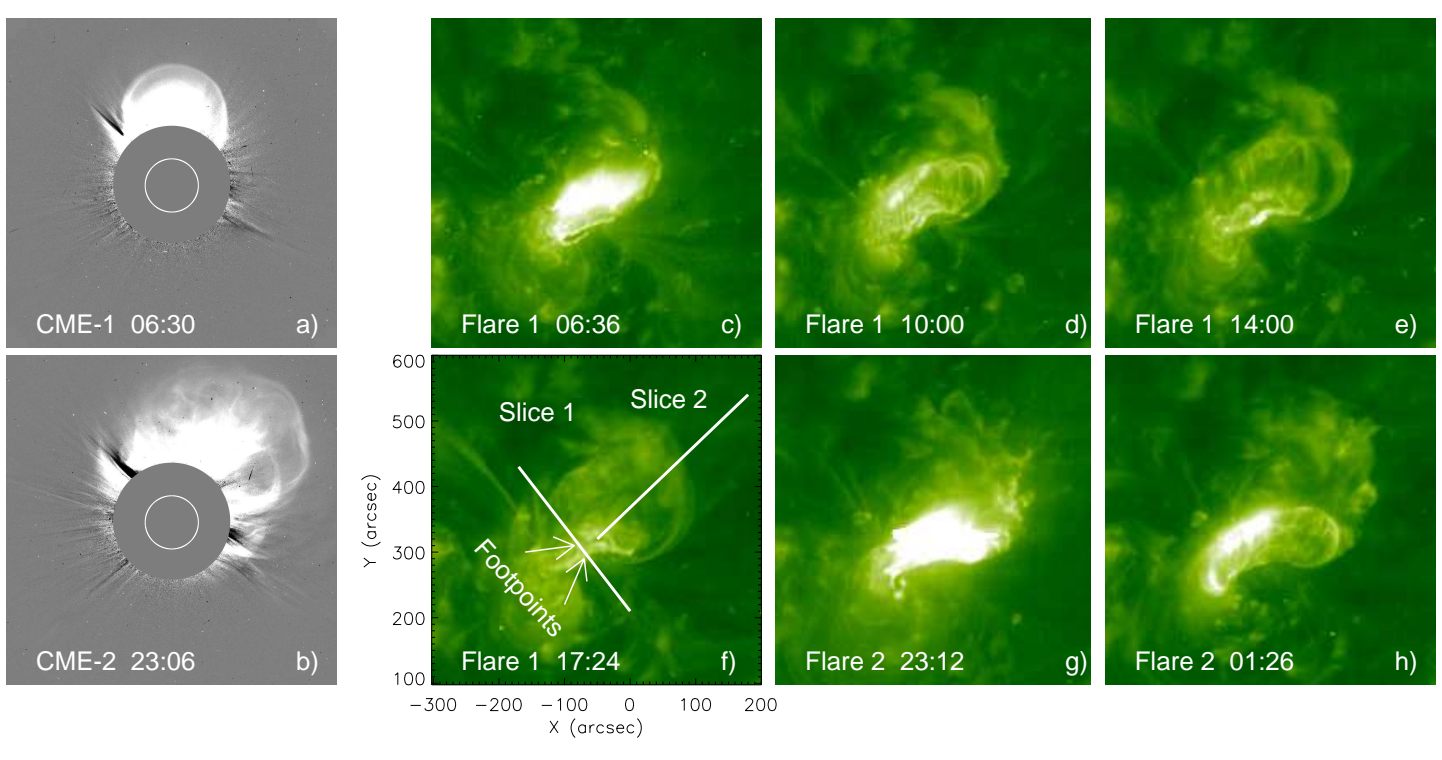

Fig. 1.- (a-b) Running difference images of CMEs 1 and 2 observed by LASCO. (c) EIT $195 \AA$ image of the CME-associated Flare 1. (d-f) Three images showing the post-flare loops after the peak time of flare 1. Slices 1 and 2 in panel (f) are used to trace the time evolution of the post-flare loop system. (g) EIT $195 \AA$ image of the CME-associated flare 2. (h) Image showing the post-flare loops of flare 2 .

(Animation of the post-flare loops is available in the online journal)

\section{Observations and Data Analysis}

On 2005 January 15, there were two halo CMEs (CME 1 and CME 2) that were observed by the C2 and C3 coronagraphs of the Large Angle and Spectrometric Coronagraph (LASCO) (Brueckner et al. 1995) on board the Solar and Heliospheric Observatory (SOHO). They first appeared in the $\mathrm{C} 2$ field of view (FOV) at 06:30 UT and 23:06 UT, respectively, as shown in Figure 1a and 1b. Their linear fitting speed 11 in the FOV of LASCO both exceeded $2000 \mathrm{~km} \mathrm{~s}^{-1}$. After visually inspecting the Extreme-ultraviolet Imaging Telescope (EIT; Delaboudinière et al. 1995) $195 \AA$ data, we find that the two extremely fast CMEs were associated with two major long-decay flares (Flare 1 and Flare 2), respectively, as seen in Figure 1c and 1g. The most important finding is that the post-flare loop system of flare 1 was always expanding and rising during the period between the two flares until part of it was activated as the second CME/flare eruption, as denoted in Figure 1c-g. The overall properties of the two CMEs and associated flares are summarized in Table 1. The kinematics of the post-flare loop system will be studied in the next section.

Inspecting the magnetic field data, we find that the two flare-associated CMEs originated from the active region NOAA 10720, which appeared on 2005 January 10 and then grew rapidly before it

\footnotetext{
${ }^{1}$ http://cdaw.gsfc.nasa.gov/CME_list
} 
was nearly at the disk center on 2005 January 10, and finally decayed on January 20. The Michelson Doppler Imager (MDI; Scherrer et al. 1995) magnetograms with 96 min cadence provides the lineof-sight magnetic field evolution of this active region. Fortunately, vector magnetic field data with a higher cadence of $\sim 1 \mathrm{~min}$ and a higher spatial resolution of $\sim 0.6^{\prime \prime}$ were obtained by the Digital Vector Magnetograph (DVMG) at BBSO on January 15, which covered a FOV of $\sim 300^{\prime \prime} \times 300^{\prime \prime}$. The sensitivity of the line-of-sight and transverse magnetic field is 2 and $20 \mathrm{G}$, respectively. Due to the filter observation, the data are saturated when the field strength exceeds $\sim 1000 \mathrm{G}$. We fill these saturated areas with the co-aligned MDI line-of-sight magnetic field to reduce its influence on the extrapolation. The projection effect is negligible since the vector magnetic field was observed at the disk center. More details about the DVMG data can be found in Jing et al. (2009). Finally, we resolve the $180^{\circ}$ azimuthal ambiguity of the transverse magnetic field using the minimum energy algorithm (Metcalf 1994; Metcalf et al. 2006), which simultaneously minimizes both the electric current density and the magnetic field divergence. This state-of-art automatic algorithm gives a satisfactory resolution of the $180^{\circ}$ ambiguity, where the changes of the field directions are smooth and the field divergence is sufficiently small in the FOV of the DVMG.

Based on the DVMG data, we reconstruct the three-dimensional (3D) coronal magnetic field by the NLFFF model using the optimization algorithm; the model was originally proposed by Wheatland et al. (2000) and implemented by Wiegelmann (2004). To deal with the inconsistency between the forced photospheric magnetic field and the force-free assumption of the NLFFF models, as well as the noises of the observed magnetic field, a preprocessing procedure was proposed by Wiegelmann et al. (2006). The preprocessing procedure minimizes the net force and torque of the photospheric magnetic field and keeps the consistency between the final preprocessed data and the measured data, given that the magnetic flux is balanced in the FOV. However, the flux balance condition is often not satisfied because of the observational limitation. Guo et al. (2010) showed that applying the preprocessing procedure on the magnetic field in the original FOV is better than embedding it in a larger FOV where the transverse components of the magnetic field are unknown. Experiments of embedding vector magnetograms into line-of-sight magnetograms have been done in De Rosa et al. (2009). It was found that the missing transverse magnetic field information usually lead to inconsistent boundary conditions. Finally, the preprocessed data are submitted to the optimization method as the bottom boundary to extrapolate the NLFFF.

Table 1: Properties of the two CME/flare events on 2005 January 15.

\begin{tabular}{ccccccccc}
\hline \hline CME & $\begin{array}{c}\text { Time }^{\text {a }} \\
(\mathrm{UT})\end{array}$ & $\begin{array}{c}\text { Speed } \\
\left(\mathrm{km} \mathrm{s}^{-1}\right)\end{array}$ & $\begin{array}{c}\text { Width } \\
(\mathrm{deg})\end{array}$ & Flare & $\begin{array}{c}\text { Onset } \\
(\mathrm{UT})\end{array}$ & $\begin{array}{c}\text { Peak } \\
(\mathrm{UT})\end{array}$ & $\begin{array}{c}\text { End } \\
(\mathrm{UT})\end{array}$ & Magnitude \\
\hline CME 1 & $06: 30$ & 2049 & 360 & Flare 1 & $05: 54$ & $06: 38$ & $07: 17$ & M8.6 \\
CME 2 & $23: 06$ & 2861 & 360 & Flare 2 & $22: 25$ & $23: 02$ & $23: 31$ & X2.6 \\
\hline
\end{tabular}

a Time of the first appearance in $\mathrm{C} 2$. 


\section{Expansion of Post-flare Loops and Magnetic Field Evolution}
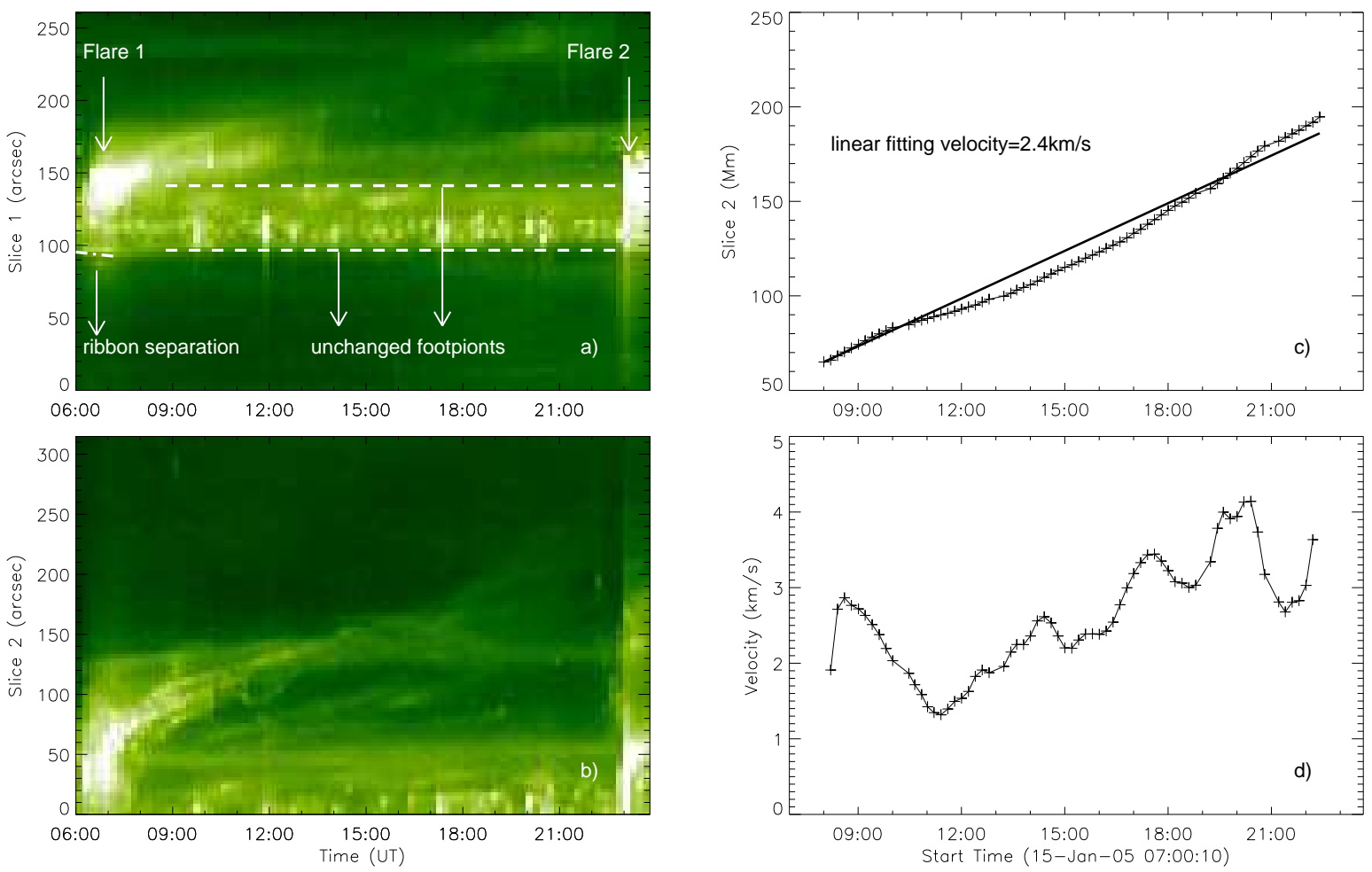

Fig. 2.- (a-b) Time evolutions of brightness along slices 1 and 2 as shown in Figure 1f. The dash-dotted and dash lines in panel (a) denote the separation of the southern flare ribbon and unchanged footpoints, respectively. (c-d) Projected height and speed evolution at the top position of the post-flare loops.

In the standard flare model, ascending of the reconnection site in the corona causes the apparent rising of the post-flare loops and the flare ribbon separation (e.g., Kopp \& Pneuman 1976). In fact, this is the manifestation of the successive stacking of reconnected loops but not the real motion of the same loop. In this study, however, we observed the real rising of a group of post-flare loops with their footpoints keeping unchanged that were then activated as the second CME. To identify that, we trace the progress of the post-flare loops and their footpoints using two slices, one of which passes through the projected top and the other crosses the footpoints of the post-flare loops, as shown in Figure 1f. The left two panels of Figure 2 show the time evolution of the brightness along slices 1 and 2, respectively. From the time evolution of slice 1, we find that during the first flare, the southern flare ribbon had an separation, as indicated by the dash-dotted line; about $1 \mathrm{hr}$ after the peak of the first flare, the footpoints of the post-flare loops kept largely unchanged until the occurrence of flare 2, as indicated by the two dash lines. Note that, the northern flare ribbon was sheltered by the post-flare loops so that it was difficult to see. Nevertheless, we can obtain 
a footpoint separation speed of $0.06 \mathrm{~km} \mathrm{~s}^{-1}$ if the uncertainty in the footpoint identification is estimated to be $5.2^{\prime \prime}$ ( 2 pixels) during the period of $\sim 16 \mathrm{hr}$. Such a speed is far less than the typical separation speed of flare ribbons of $10 \mathrm{~km} \mathrm{~s}^{-1}$ (Qiu et al. 2002; Jing et al. 2005), which confirms that the footpoints did not change. On the other hand, from the time evolution of slice 2, one can notice that the top of the post-flare loops was continually rising. We then measure the height-time variation of the top position along slice 2, as shown in the right upper panel of Figure 2. The linear fitting speed is $2.4 \mathrm{~km} \mathrm{~s}^{-1}$. The deprojected speed is $\sim 8 \mathrm{~km} \mathrm{~s}^{-1}$ if we assume that the loops rose radially, which is far more than the maximal rising speed caused by the ascending reconnection site of $\sim 1.1 \mathrm{~km} \mathrm{~s}^{-1}$ by Hick \& Svestka (1985). Furthermore, through the piecewise numerical derivative method, the velocity evolution can be obtained from the height-time measurement (Figure $2 \mathrm{~d}$ ). We find that the rising of the post-flare loops was further accelerating, but not decelerating, after 12:00 UT until the occurrence of flare 2. Based on the standard flare model and the observations of Švestka (1996), a rising motion of post-flare loops with a decelerated or constant speed can be regarded as an apparent motion resulting from the ascending reconnection site. In this letter, however, we report a rising motion of the post-flare loops with an accelerating speed that we think represents a real ascending motion of the loops through the corona.

We believe that the rising of the post-flare loops was driven by the magnetic flux emergence and the rotation of the sunspot. The evolution of the magnetic flux of the active region NOAA 10720 from January 13 to 17 is plotted in Figure 3a. The two vertical lines denote the onset time of flares 1 and 2. We note that the total unsigned magnetic flux was continually increasing after the occurrence of flare 1, which was mainly due to the rapid emerging of the negative flux. We also plot two MDI line-of-sight magnetograms around the onset time of the two flares in Figure 3b and 3c. After comparing the two images, we find that the PIL of the bipolar active region was elongated about $20^{\prime \prime}$ due to the westward motion and rotation of the western sunspot with a negative polarity, as indicated by the arrows in Figure $3 \mathrm{~b}$ and 3c. Note that, the motion and rotation of the negative polarity may be driven essentially by the horizontal plasma flows in the photosphere. Usually, we know that the magnetic free energy tends to decrease after the occurrence of a major flare. Though, in some cases, one eruption does not necessarily release the entire free energy of the active region, as revealed in Thalmann \& Wiegelmann (2008), who studied the time-series of a NLFFF-equilibria computed from SOLIS. For the event in this study, the continual magnetic flux emergence and the rotation of the sunspot enhanced the magnetic pressure under the post-flare loops and thus drove their rising and expanding motion; in the meantime, they increased again the magnetic free energy in the corona. The continually increasing magnetic free energy, in spite of the part that was released during the first eruption, was still enough for the second eruption of CME/flare.

Another interesting finding is that the flux rope-like structure remained to exist after the eruption of the first CME, which continually ascended prior to the second CME. With the observed vector magnetic field in the photosphere, we extrapolate the $3 \mathrm{D}$ NLFFF configuration of this active region. The field lines are plotted in Figure 4. The figure shows that the flux rope was under significant development during the time period. It kept rising with its length and twist 

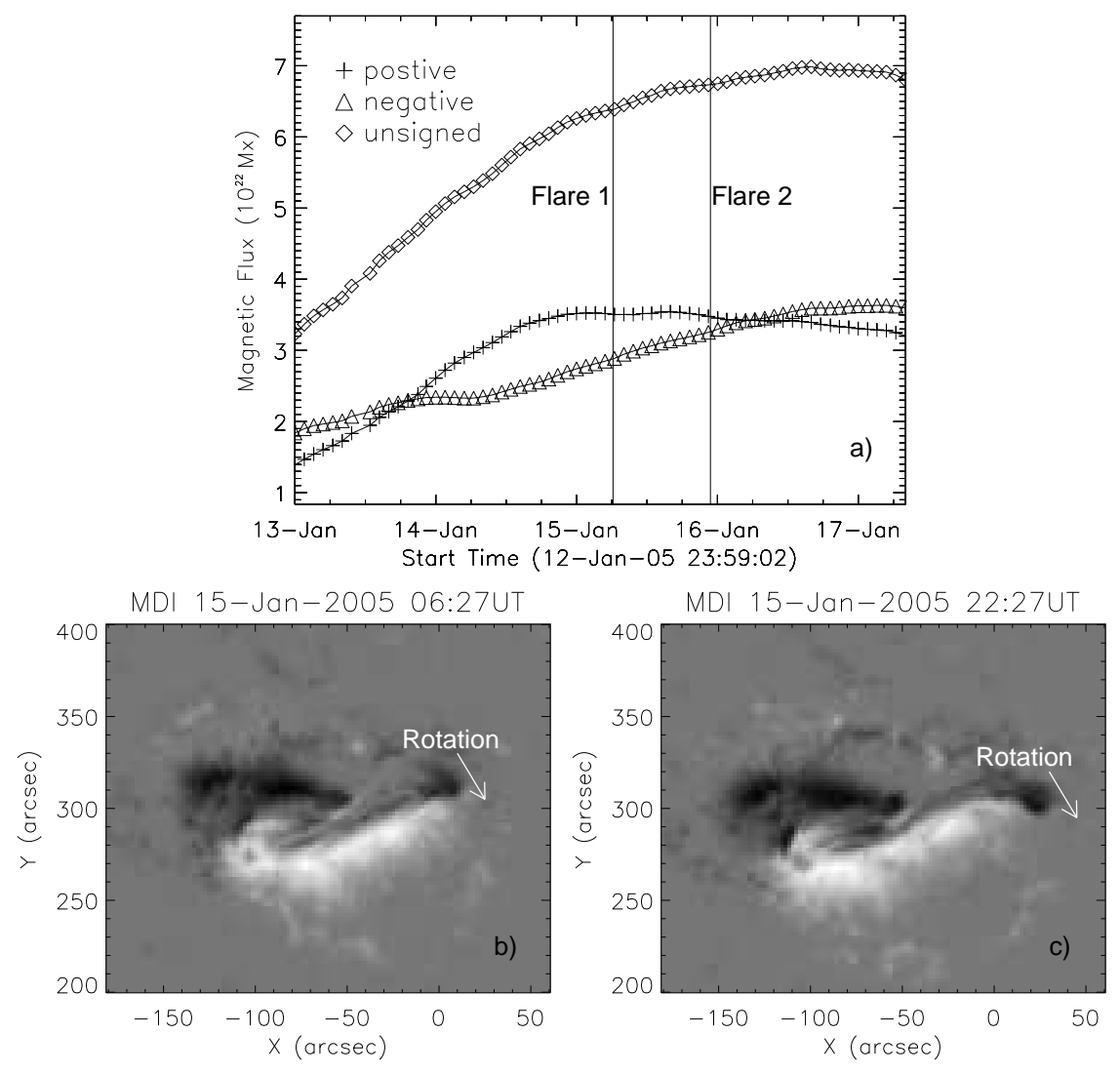

Fig. 3.- (a) Evolution of the magnetic flux for the whole FOV of the active region. The two vertical lines denote the onset times of the two flares. (b-c) Line-of-sight magnetograms with a field of view of $240^{\prime \prime} \times 200^{\prime \prime}$ taken from MDI. The two arrows show the motion direction of the western sunspot with a negative polarity.

increasing. In particular, the top of the flux rope rose from $\sim 13^{\prime \prime}$ at 18:00 UT to $\sim 17^{\prime \prime}$ at 23:00 UT. The rising of the flux rope may provide a strong evidence of flux rope emergence through the photosphere into the corona. In addition, the developed flux rope may also have a role in enhancing the magnetic pressure underneath the post-flare loop systems, which tends to push the loop system upward. It is noteworthy that whether the flux rope exists in CME/flare producing regions is still an open and unresolved issue. Up to now, there are only a few studies that showed the extrapolated flux rope based on the vector magnetic field observation in the photosphere (e.g., Yan et al. 2001; Canou et al. 2009; Guo et al. 2010). However, in this letter, we not only obtain a flux rope structure with a strong twist but also its development. The detailed evolution of the flux rope obtained by the DVMG vector field data with a high cadence will be described in a future paper. 

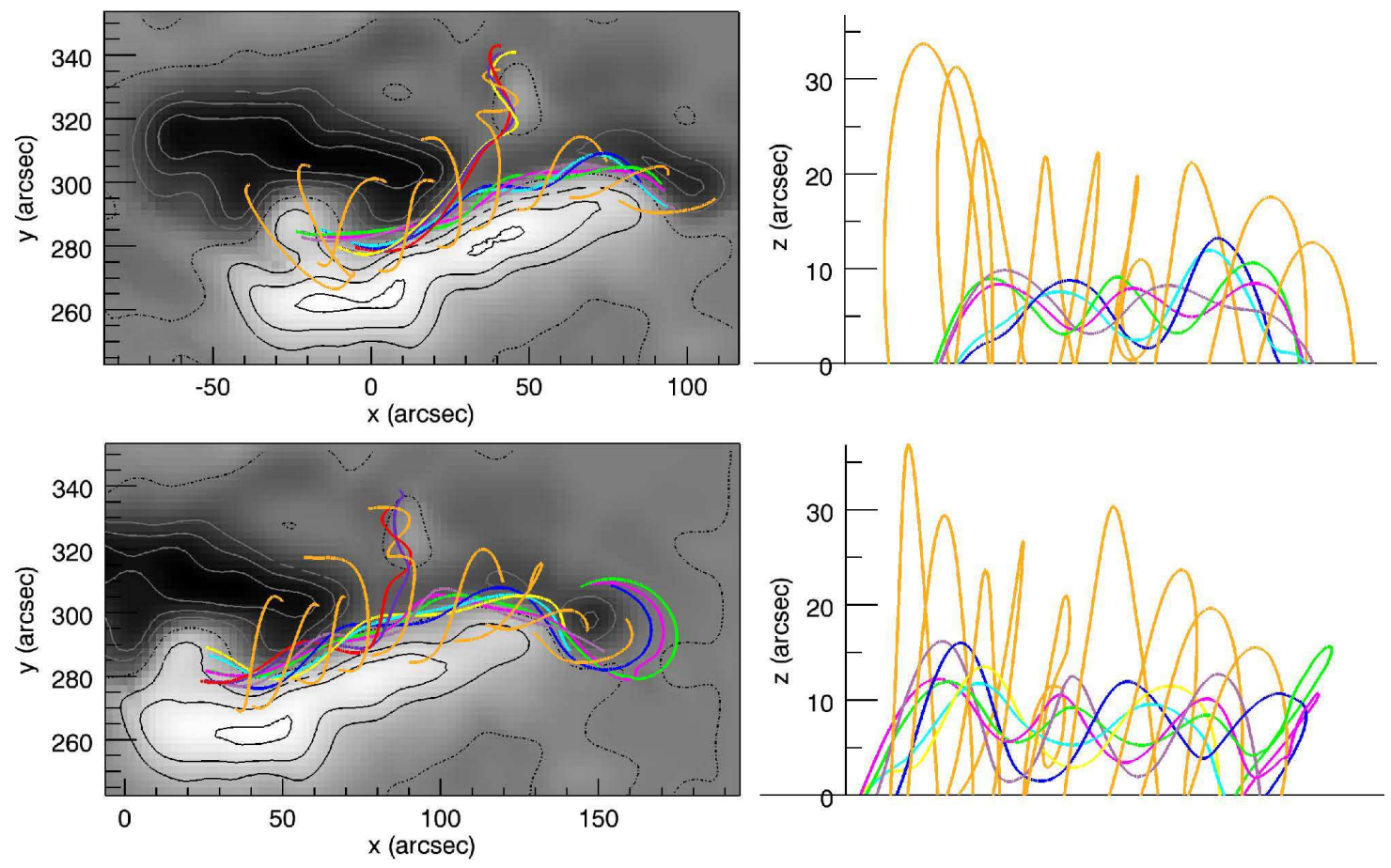

Fig. 4. - Top view (a) and edge-on view (b) of the extrapolated 3D magnetic field configuration at 18:00 UT on 2005 January 15. Panels (c) and (d) are like (a) and (b), respectively, but for the time of 23:00 UT on 2005 January 15. In panels (a) and (c), the background image is the processed line-of-sight magnetogram. Note that the scale in $z$-direction is three times that in $x$ and $y$-directions; the smaller flux rope in the northern branch in panels (a) and (c) are not plotted in panels (b) and (d).

\section{Summary and Discussions}

In this study, we report the full evolution of a post-flare loop system, characterized by the initial rising and expanding motion and the final re-activation as another CME. We find that the rising and expanding of the post-flare loops were essentially due to the continual increase of the magnetic flux, which enhanced the magnetic pressure underneath the post-flare loop system. On the other hand, we speculate that the first CME/flare eruption may lead to a weakening of the constraining tension force of the overlying field to the post-flare loop system. We further study the development of the associated flux rope by the NLFFF extrapolation, and find that the twist and length of the flux rope were increased, which may be due to the continual flux emergence and/or the rotation of the sunspot. In fact, magnetic flux emergence (Fan 2001; Fan \& Gibson 2003, 2004) and sunspots rotation caused by photospheric plasma flows (Amari et al. 2000; Török \& Kliem 2003, 2005) are among the most plausible ways that have been invoked in numerical simulations to increase the magnetic non-potentiality leading to the final eruption. 
Through a quasi-linear force free method (Wang et al. 2001), Zhao et al. (2008) studied the magnetic topology skeleton of this active region. They found the rising of the magnetic null and its spine, which is consistent with the rising of the extrapolated flux rope and the strong twist of the flux rope as shown here. This strongly twisted flux rope provides a source for the shear of the post-flare loop system that enhances the non-potentiality of associated magnetic configuration. Jing et al. (2009) studied the temporal evolution of the magnetic free energy for this active region from 21:00 to 23:00 UT on January 15 and found that it tends to increase prior to the onset of flare 2. The accumulation of the free energy was mainly caused by the emergence of magnetic flux and the rotation of the sunspots, which continually transfers energy below the photosphere into the corona (Kurokawa et al. 2002; Cheng et al. 2010b). Therefore, the post-flare configuration may still accumulate magnetic free energy in a short time that is responsible for the second eruption.

It is worthy noting that the homologous flares can take place in the same active region with similar morphologies and pattern of development (e.g., Choe \& Cheng 2000), and the successive flares usually occur in the magnetic loops that are closely related but not in exactly the same loops (e.g., Liu et al. 2009). Whereas, the most significant difference, of the events in this studies from the general homologous and successive flares, is that the second CME/flare came directly from the post-flare loops of the first eruption but not the loops structures in the similar or other related locations.

In conclusion, it is commonly viewed that a post-flare loop system evolves relatively uneventful after their formation following a CME/flare eruption. Except for some dynamic flows along the post-flare loops (e.g., Brekke et al. 1997; Qiu et al. 1999), the magnetic structure of the loops is closer to potential than that before the eruption, thus unable to re-erupt in a short time after a major eruption. The event studied here provides an exceptional case and may break such a point of view. It shows that a post-flare magnetic configuration can evolve quickly to a state that is favorable for another major CME/flare eruption, as long as there is continual emergence of flux rope and/or rotation of sunspots. Post-flare loops can re-flare in a short time. Models and prediction of solar flares (CMEs) should take into account such an observational fact.

We thank the anonymous referee for his/her valuable comments that helped to improve the paper. X.C., M.D.D., and Y.G. were supported by NSFC under grants 10673004, 10828306, and 10933003 and NKBRSF under grant 2006CB806302. J.Z. was supported by NSF grant ATM0748003 and NASA grant NNG05GG19G. J.J. was supported by NSF under grant ATM 09-36665 and ATM 07-16950. T.W. was supported by DLR-grant 50 OC 0501. SOHO is a project of international cooperation between ESA and NASA.

\section{REFERENCES}

Amari, T., Luciani, J. F., Mikic, Z., \& Linker, J. 2000, ApJ, 529, L49 
Aulanier, G., Török, T., Démoulin, P., \& DeLuca, E. E. 2010, ApJ, 708, 314

Brekke, P., Kjeldseth-Moe, O., \& Harrison, R. A. 1997, Sol. Phys., 175, 511

Brueckner, G. E., et al. 1995, Sol. Phys., 162, 357

Bruzek, A. 1964, ApJ, 140, 746

Canou, A., Amari, T., Bommier, V., Schmieder, B., Aulanier, G., \& Li, H. 2009, ApJ, 693, L27

Carmichael, H. 1964, NASA Special Publication, 50, 451

Cheng, X., Zhang, J., Ding, M. D., \& Spoomvises, W. 2010a, ApJ, 712, 752

Cheng, X., Ding, M. D., \& Zhang, J. 2010b, ApJ, 712, 1302

Choe, G. S., \& Cheng, C. Z. 2000, ApJ, 541, 449

Delaboudinière, J.-P., et al. 1995, Sol. Phys., 162, 291

De Rosa, M. L., et al. 2009, ApJ, 696, 1780

Fan, Y. 2001, ApJ, 554, L111

Fan, Y., \& Gibson, S. E. 2003, ApJ, 589, L105

Fan, Y., \& Gibson, S. E. 2004, ApJ, 609, 1123

Guo, Y., Schmieder, B., Démoulin, P., Wiegelmann, T., Aulanier, G., Török, T., \& Bommier, V. 2010, ApJ, 714, 343

Harra-Murnion, L. K., et al. 1998, A\&A, 337, 911

Hirayama, T. 1974, Sol. Phys., 34, 323

Hick, P., \& Svestka, Z. 1985, Sol. Phys., 102, 147

Jing, J., Qiu, J., Lin, J., Qu, M., Xu, Y., \& Wang, H. M. 2005, ApJ, 620, 1085

Jing, J., Chen, P. F., Wiegelmann, T., Xu, Y., Park, S.-H., \& Wang, H. 2009, ApJ, 696, 84

Kahler, S. 1977, ApJ, 214, 891

Kliem, B., \& Török, T. 2006, Physical Review Letters, 96, 255002

Kopp, R. A., \& Pneuman, G. W. 1976, Sol. Phys., 50, 85

Kurokawa, H., Wang, T., \& Ishii, T. T. 2002, ApJ, 572, 598

Liu, C., Lee, J., Karlický, M., Prasad Choudhary, D., Deng, N., \& Wang, H. 2009, ApJ, 703, 757 
Metcalf, T. R. 1994, Sol. Phys., 155, 235

Metcalf, T. R., et al. 2006, Sol. Phys., 237, 267

Metcalf, T. R., et al. 2008, Sol. Phys., 247, 269

Pick, M., et al. 2006, Space Science Reviews, 123, 341

Qiu, J., Lee, J., Gary, D. E, \& Wang, H. 2002, ApJ, 5651335

Qiu, J., Wang, H., Chae, J., \& Goode, P. R. 1999, Sol. Phys., 190, 153

Schrijver, C. J., et al. 2008, ApJ, 675, 1637

Schrijver, C. J. 2009, Advances in Space Research, 43, 739

Scherrer, P. H., et al. 1995, Sol. Phys., 162, 129

Sturrock, P. A. 1966, Nature, 211, 695

Su, Y., van Ballegooijen, A., Schmieder, B., Berlicki, A., Guo, Y., Golub, L., \& Huang, G. 2009, ApJ, 704, 341

Švestka, Z. 1996, Sol. Phys., 169, 403

Thalmann, J. K., \& Wiegelmann, T. 2008, A\&A, 484, 495

Török, T., \& Kliem, B. 2003, A\&A, 406, 1043

Török, T., \& Kliem, B. 2005, ApJ, 630, L97

Wang, H., Yan, Y., \& Sakurai, T. 2001, Sol. Phys., 201, 323

Wheatland, M. S., Sturrock, P. A., \& Roumeliotis, G. 2000, ApJ, 540, 1150

Wiegelmann, T. 2004, Sol. Phys., 219, 87

Wiegelmann, T., Inhester, B., \& Sakurai, T. 2006, Sol. Phys., 233, 215

Yan, Y., Deng, Y., Karlický, M., Fu, Q., Wang, S., \& Liu, Y. 2001, ApJ, 551, L115

Zhao, H., Wang, J.-X., Zhang, J., Xiao, C.-J., \& Wang, H.-M. 2008, Chinese Journal of Astronomy and Astrophysics, 8, 133 